\title{
ON THE APPLICATION OF STOCHASTIC OPTIMAL CONTROL TO PENSION FUND MANAGEMENT
}

\author{
Bright O.Osu ${ }^{1}$ and Onwukwe ljioma ${ }^{2}$ \\ ${ }^{1,2}$ Mathematics Department, Abia State University, Uturu, Nigeria.
}

\begin{abstract}
Keywords: Stochastic Optimal control, Pension fund Management, Defined Contribution, Defined Benefit.
\end{abstract}

1. Abstract In this paper, we apply the stochastic optimal control theory to the pension fund management before and after retirement in the defined contribution and defined benefit pension schemes, where benefits are paid as investment returns for a period or duration of time. The goal of the management problem is to optimize the long-term growth of expected utility of returns. We consider different types of power law utility function of the form $U(X)=\gamma^{-1} X^{\gamma}, \gamma<1, \gamma \neq 0$ to examine the different investment schemes. Our result shows the advantage of the defined contribution scheme over the defined benefit scheme before and after retirement.

\section{Introduction}

The issue of selecting the appropriate investment scheme for pension managers is becoming more challenging especially for defined contribution scheme.

Notable contributions have been made in this area especially in the use of classical tools as portfolio theory (Merton, 1969), Optimal control (Merton, 1971; Deelstra et al., 2000; Cairns, 2000; Menoncin, 2002), with consequences of a stochastic environment (Boulier et al., 2000).

On the other hand, Fleming (1995) reformulated optimal investment models as risk-sensitive stochastic control problem. This approach was used in Fleming and Sheu (1999) to give a detailed analysis of an investment analysis of an investment model in which only one risky and riskless asset are considered and transaction are ignored. Meanwhile, Constantinides $(1979,1986)$ had shown that the optimal transaction policy is to maintain the ratio of the dollar amount invested in the riskless asset to that in the risky asset within a certain range, represented by the buy boundary and sell boundary. Consequently three regions are identified depending on the portfolio ratio: the notransaction region, the buy region and the sell region.

Instead in this paper, we consider a situation where a pensioner relies on a fund manager for access to risky investment. As in Wang and Wang(2011), we characterise optimal fund management by the fund manager who receives a management fund that is paid over time period at a fraction of current assets under management. The fund manager accesses the fund via defined contribution or defined benefit.

A defined contribution plan is a scheme where only contributions are fixed and benefits depends on the returns on the assets of the funds. The risk derived from the fund management is borne by the beneficiary. This is unlike to the defined benefit plan where the benefits are normally related to the final salary level and the financial risk is assumed by the sponsor agent. The main objective of the shareholder in the defined contribution plan is to maximize the expected utility obtained from fund accumulation at a fixed date $t$. The contribution rate $\alpha(t)$ is exogenous to this optimization process, since it is generally determined by salary.

Using the method in Oksendal, 1998), we investigate herein the application of stochastic optimal control as optimization techniques that will guarantee suitable life styling before and after retirement (given defined contribution and define benefit) by finding its explicit solutions using some utilities functions such as power law utility of the form $U(X)=\gamma^{-1} X^{\gamma}, \gamma<1, \gamma \neq 0$ and interpreting the solutions using a derived process ${ }^{Z_{t}^{\prime}}$ which can be recast in terms of an asset and liability model(ALM) as in $\operatorname{Osu}(2011)$ 


\section{Formulation of the Pension fund management investment Problems:}

We consider a defined contribution pension scheme where benefits are paid from investment returns span over a period of time horizon $\mathrm{T}$. we are faced with the problem of finding the optimal investment policy of the contributions (assets) that will guarantee pension liabilities during the life span of the participant before and after retirement.

The financial market available for the portfolio allocation problem by the pension fund manager is the Black-Scholes model (see Bjoik, 1998). This consists of two assets - riskless one, whose price process $S_{t}{ }^{o}$ is governed by the dynamics

$$
d S_{t}^{o}=r S_{t}^{o} d t
$$

Where $r>0$, and a risky asset, whose price dynamics $S_{t}$ follows a geometric Brownian motion

$$
d S_{t}=\mu S_{t} d t+\sigma S_{t} d W_{t}
$$

with drift $\mu>0$ and diffusion $\sigma>0$ are some given constants, where $W_{t}$ is a standard Brownian motion defined on a complete filtered probability space $\left(\Omega, f,\left(f_{t}\right), P\right)$ with $f_{t}=\sigma\left\{W_{s}: s \leq t\right\}$. The investment policy is defined by a $F$-adapted process $\pi=\left\{\pi_{t},[0, T]\right\}$ where $\pi_{t}$ represents the proportion of pension fund contributed within the time $t$ invested in the riskless asset at time $t$; the remaining $\left(\left(1-\pi_{t}\right)\right.$ proportion of the pension fund or wealth accumulated is invested in the risky asset.

Therefore, the wealth process satisfies

$$
\begin{aligned}
& d X_{t}=\pi_{t} X_{t} \frac{d S_{t}}{d t}+\left(1-\pi_{t}\right) X_{t} \frac{d S_{t}^{0}}{S_{t}^{0}} \\
& X_{s}=X_{t} \exp \left(r(s-t)+(\mu-r) \int_{t}^{s} \pi_{t} d t-\frac{1}{2} \sigma^{2} \int_{t}^{s} \pi_{t}^{2} d t+\sigma \int_{t}^{s} \pi_{t}\right)
\end{aligned}
$$

such a process

$$
\pi \text { is said to be admissible if } E\left[\int_{0}^{T}|\pi|^{2} d t\right]<\infty
$$

The liabilities after retirement are to be paid from the returns on investments by the funds manager. During the phase of activity period, the funds can be invested in riskless or risky assets; the reserve obtained at retirement age $(T)$ is the portion of wealth accumulated without any guarantee given by the fund manager.

The fund manager is faced with the problem of investing the reserve in risky assets.

We have stated the dynamics of the process from equations (1) to (4).

We consider the problem in three stages, due to the presence of liability only after retirement.

[1]: consider the pre-retirement $(t \in[0, N])$, a period without liability; we are

only faced with optimizing the utility of the final wealth. Consider the price process of (1) and (2), the process $S$ is the solution of the stochastic differential equation with $S(0)=p=$ lump sum paid at time $t_{0}$

[2]: consider the time of retirement $(t=N)$, the participant is about to exit from contributing to the fund. The fund manger will consider how to invest and what proportion to be paid to the participant and to invest.

[3]: consider the post-retirement $(t \in[N, N+T])$, the fund process is given by the SDE, where a proportion of the fund is invested in risky asset

$$
d X_{t}=X_{t}\left\lceil\left\lfloor\left(r+(\mu-r) \pi_{t}\right) d t+\sigma \pi_{t} d W_{t}\right\rceil\right\rfloor
$$


Let $X_{t}$ denote the wealth at time $t$ of some fund invested in the financial market. We assume that the investor allocates continuously his wealth between the riskless and risky assets.

We shall denote by $\pi^{i}$ the proportion of the pension funds invested in the $i^{\text {th }}$ risky asset. This means that $\pi_{t}^{i} X_{t}$ is the amount invested at time $\mathrm{t}$ in the $i^{t h}$ risky asset, the remaining proportion of wealth $1-\sum_{i=1}^{d} \pi_{t}^{i}$ is invested in the non-risky assets.

The self-financing condition states that the variation of the wealth is only affected by the variation of the price process. Under this condition, the wealth process satisfies

$$
\begin{aligned}
& \qquad \begin{aligned}
d X_{t} & =X_{t} \sum_{i=1}^{d} \pi_{t}^{i} \frac{d S_{t}^{i}}{S_{t}^{i}} \\
& =X_{t} \pi_{t}^{l}\left[b_{t} d t+\sigma_{t} d W_{t}\right] \\
& =X_{t} \pi_{t}^{i} \sigma_{t} d W_{t}^{0}
\end{aligned} \\
& \qquad \begin{array}{l}
X_{T}^{x, \pi}=x \exp \left(\int_{0}^{t} \pi_{r}^{l} \sigma_{r} d W_{r}^{0}\right), 0 \leq t \leq T
\end{array}
\end{aligned}
$$

In the pension asset allocation process, the fund manager faces some randomness. Let $\pi_{t}$ and $1-\pi_{t}$ be the proportion of pension funds invested in the money market stock.

We supposed that $\left\{\left(\pi_{t}, 1-\pi_{t}\right): t>0\right\}$ is a Markovian control adopted to the filtration $\left\{f_{t}\right\}_{t z 0}$ and satisfying (see Josa-Fombellida and Rincon Z. (2010))

$$
E \int_{0}^{t}\left(\pi_{t}^{2}+\left(1-\pi_{t}\right)^{2}\right) d t<\infty
$$

\section{DEFINITION: 1}

An investment strategy is a $F$-adapted process $\pi$ valued in $\mathrm{R}^{\mathrm{n}}$ and satisfying

$$
\int_{0}^{T}\left|\sigma_{t}^{1}\right|^{2} d t<\infty \quad p-a . s . X^{x, \pi} \text { is a } p^{0}
$$

martingale under $\mathrm{p}^{\mathrm{o}}$

super martingale, (7) as a non-negative local We are faced with the problem of optimizing the expected utility of the final wealth at the end of the periods.

i.e. maximization of the expected utility of the total pension funds obtained at retirement age.

$$
\underset{u}{\operatorname{Max}} \operatorname{EU}(X(N))
$$

Maximization of the expected utility of the surplus after payment of pension during $\mathrm{T}$-periods is defined as;

$$
\operatorname{Max}_{u} E U(X(N+T))
$$

We are interested in the investigating the optimal policies in the two periods. 


\section{DEFINITION: 2}

A progressively measurable, self-financing portfolio strategy $\pi($.$) with initial wealth$ say $x>0$ is called admissible if $X(t) \geq 0$ a.s. for $0 \leq t \leq T$ (see Lim and Wong (2010))

Suppose that a participant decides to put a constant proportion of salary into personal pension fund. Then the defined contribution level related to this is as follows (see Deelstra,G., and Koehl (2002)) $c(t)=c(0) e^{i t} \forall t \in[0, T]$ Wages are subject to increase continuously at the inflation rate $i$

\section{OPTIMAL POLICY BEFORE RETIREMENT WITH CONTRIBUTION RATE}

We are required to solve equation (8), that is

\section{$\operatorname{Max}_{u} E U(X(N))$ with}

$$
d X_{t}=X_{t}\left[U_{t} \alpha+\left(1-U_{t}\right) r\right] d t+X_{t} U_{t} \sigma d W_{t}, X(0)=p(0 \leq t \leq N)
$$

Where $\alpha$ is the contribution rate of the pensioner.

We state the value function of the problem as

$$
W(t, X)=\operatorname{Max}_{u} E[U(X(N)) \mid X(t)=X]
$$

with its associated HJB equation

$$
0=\max _{\{u\}}\left[\frac{\partial w}{\partial t}+\left(u_{t}(\alpha-r)+r\right) X \frac{\partial w}{\partial x}+\frac{1}{2} u_{t}^{2} \sigma^{2} X^{2} \frac{\partial^{2} w}{\partial x^{2}}\right]
$$

The pension fund manager objective is to choose an allocation of the wealth so as to maximize the expected utility of the terminal wealth, we have

$$
V(t, x)=\operatorname{Sup}_{S \in R_{+}^{2}} E_{t, x}[U(X)]
$$

with the HJB equation associated with the problem and Uis the set of all admissible portfolios, is a modified equation of (12). ( Osu, B.O.(2011))

$$
\frac{\partial w}{\partial t}(t, x)+\operatorname{Sup}_{S \in R^{2}} L^{u} W(t, x)=0
$$

Where $\mathrm{L}^{\mathrm{u}}$ is the $2^{\text {nd }}$ order linear operator

$$
\begin{aligned}
& L^{u} W(t, x)=\left(U_{t}(\alpha-r)+r\right) X \frac{\partial w}{\partial x}(t, x)+\frac{1}{2} \sigma^{2} \alpha^{2} x^{2} \frac{\partial^{2} w}{\partial x^{2}}(t, x) \\
& \text { Put (12) as } 0=\max _{\{u\}}\{\lambda\} \\
& \qquad \text { so that } E_{t, x}\left[U\left(X_{T}\right)\right]=x^{r} E_{t, 1}\left[U\left(X_{T}\right)\right] \text { and } \\
& \qquad V(t, x)=x^{r} V(t, 1)
\end{aligned}
$$

Also from (16), we can derive the following two equations and a $2^{\text {nd }}$ order condition.

$$
\left.\begin{array}{l}
\text { (a) } \lambda\left(u_{t}^{*}\right)=0 \\
\text { (b) } \frac{\partial \lambda}{\partial u}\left(u_{t}^{*}\right)=0 \\
\text { (c) } \frac{\partial^{2} \lambda\left(u_{t}^{*}\right)}{\partial u^{2}}<0
\end{array}\right\}
$$


Plug back into HJB in (12), we have

$$
0=(\alpha-r) X \frac{\partial w}{\partial x}+u_{t}^{*} X^{2} \sigma^{2} \frac{\partial^{2} w}{\partial x^{2}}
$$

we obtain a first explicit form for the optimal investment proportion ${ }^{u_{t}^{*}}$ in risky asset

$$
u_{t}^{*}=-\frac{\hat{\omega} w / \alpha x}{X\left(\partial^{2} w / \partial X^{2}\right)} \bullet \frac{(\alpha-r)}{\sigma^{2}}
$$

Substitute (19) into (17) we obtain a pde for the value function

$$
\begin{aligned}
& \frac{\partial w}{\partial t}+r X \frac{\partial w}{\partial x}-\frac{1}{2} \frac{(\alpha-r)^{2}}{\sigma^{2}} \frac{(\partial w / \partial x)^{2}}{\partial^{2} w / \partial x^{2}}=0 \\
& \text { with condition } W(N, X)=U(X)
\end{aligned}
$$

We are faced with the problem of solving (20) for the value function W and substitute it in (19) to obtain the optimal policy.

This equation admits some explicit solutions for the power law utility

$$
U(X)=\frac{X^{\gamma}}{\gamma}(\text { with } \gamma<1, \gamma \neq 0)
$$

(20a) is a utility function with a constant relative aversion (CRRA)

$$
\text { (CRRA) }-\frac{U^{\prime \prime}}{U^{\prime}} X=1-\gamma
$$

The solution of (20) with the following structure

$$
\begin{aligned}
& W(t, X)=\beta_{t} \frac{X^{\gamma}}{\gamma} \text { with } \beta_{N}=1 \text { (limit condition) } \\
& \frac{\partial w}{\partial t}=\beta_{t}^{\prime} \frac{X^{\gamma}}{\gamma} \\
& \frac{\partial w}{\partial X}=\beta_{t} X^{\gamma-1} \\
& \frac{\partial^{2} w}{\partial X^{2}}=\beta_{t}(\gamma-1) X^{\gamma-2}
\end{aligned}
$$

The differential equation for

$$
\beta \text { from }(20) \text { is } \beta_{t}^{\prime}+r \gamma \beta_{t}+\beta_{t} \gamma \frac{(\alpha-r)^{2}}{2 \sigma^{2}(1-\gamma)}=0
$$

The solution is given by

$$
\beta_{t}=e^{\mu(t-N)}, \text { where } \mu=-r\left[r+\frac{(\alpha-r)^{2}}{2 \sigma^{2}(1-\gamma)}\right]
$$

$$
W(t, X)=e^{\mu(t-N)} \frac{X^{\gamma}}{\gamma}
$$

And

The $2^{\text {nd }}$-order condition is also satisfied:

$$
\frac{\partial^{2} \lambda}{\partial u^{2}}=X^{2} \sigma \frac{\partial^{2} w}{\partial X^{2}}=X^{2} \sigma^{2} e^{\mu(t-N)}(\gamma-1) X^{\gamma-2}<0
$$

using (19) the optimal investment proportion $U_{t}^{*}$ we obtained the explicit optimal policy:

$$
U_{t}^{*}=\frac{\beta_{t} X^{\gamma-1}}{X \beta_{t}(\gamma-1) X^{\gamma-2}} \frac{\alpha-r}{\sigma^{2}}, \quad U_{t}^{*}=\frac{\alpha-r}{\sigma^{2}} \cdot \frac{1}{1-\gamma}
$$

This is a constant proportion depending on the risk premium $\alpha-r$, the volatility ${ }^{2}$ and the risk aversion $^{\gamma}$ (Cairns, A., (2000)) 
OPTIMAL POLICY AFTER RETIREMENT WITH TRANSACTION COST: Using the same methodology in section 5 above, we formulate the problem as

$$
\begin{aligned}
& \max _{u} E U(X(N+T)) \\
& \text { subject to } d X_{t}=\left\{X_{t}\left[u_{t} \alpha+\left(1-\mu_{t}\right) r-\psi\right]\right\} d t+X_{t} u_{t} \sigma d W_{t},(N \leq t \leq N+Y)
\end{aligned}
$$

where $\mathrm{X}(\mathrm{N})$ is the amount obtained at retirement.

The value function of process (26) is defined by

$$
V(t, X)=\max _{u} E\left\{U(X(N+T)) \mid X_{t}=X\right\}, N \leq t \leq N+T
$$

and the maximum principal gives

$$
0=\max _{\{u\}}\left\{\frac{\partial V}{\partial t}+\left[\left\{U_{t}(\alpha-r)+r\right\} X-\psi\right] \frac{\partial V}{\partial X}+\frac{1}{2} U_{t}^{2} \sigma^{2} X_{t}^{2} \frac{\partial^{2} W}{\partial X^{2}}\right\}
$$

OR

$$
0=\max _{u}\{\theta\}
$$

We obtained the following three conditions
(a) $\theta u^{*}=0$
(b) $\frac{\partial \theta}{\partial u}\left(u^{*}\right)=0$
(c) $\frac{\partial^{2} \theta}{\partial u^{2}}\left(u^{*}\right)<0$

We have the same kind of explicit form for the optimal control as in (19)

$$
u_{t}^{*}=-\frac{\partial V / \partial X}{X\left(\partial^{2} V / \partial X^{2}\right)}-\frac{\alpha-r}{\sigma^{2}}
$$

Substitute in (29) we obtain the pde

$$
\frac{\partial V}{\partial t}+\frac{\partial V}{\partial X}(r X-\psi)-\frac{1}{2} \frac{(\alpha-r)^{2}}{\sigma^{2}} \frac{(\partial V / \partial X)^{2}}{\left(\partial^{2} V / \partial X^{2}\right)}=0
$$

\section{with $V(N+T, X)=U(X)$}

Again, we need to solve (31) for the value function Vand substitute it in (30) to obtain the optimal policy. This equation also admits some explicit solutions for power law utility of the form

$$
\begin{aligned}
U(X)= & \frac{X^{\gamma}}{\gamma}(\gamma<1, \gamma \neq 0) \\
V(t, X) & =\frac{\beta\left(X-a_{t}\right)^{\gamma}}{\gamma} \text { with limit conditions } a(N+T)=0, \beta(N+T)=1
\end{aligned}
$$

Then,

$$
\begin{aligned}
& \frac{\partial V}{\partial t}=\beta_{t}^{\prime} \frac{\left(X-a_{t}\right)^{\gamma}}{\gamma}-\beta_{t}\left(X-a_{t}\right)^{\gamma-1} a_{t}, \\
& \frac{\partial V}{\partial X}=\beta_{t}\left(X-a_{t}\right)^{\gamma-1}, \\
& \frac{\partial^{2} V}{\partial X^{2}}=\beta_{t}(\gamma-1)\left(X-a_{t}\right)^{\gamma-2}
\end{aligned}
$$


Plug back these derivatives in (31)

$$
\begin{aligned}
& \beta_{t}^{\prime} \frac{\left(X-a_{t}\right)^{\gamma}}{\gamma}-\beta_{t}\left(X-a_{t}\right)^{\gamma-1} a_{t}^{\prime}+(r X-\psi) \beta_{t}\left(X-a_{t}\right)^{\gamma-1} \\
& -\frac{1}{2} \frac{(\alpha-r)^{2} \beta_{t}^{2}\left(X-a_{t}\right)^{2(\gamma-1)}}{\sigma^{2} \beta_{t}(\gamma-1)\left(X-a_{t}\right)^{\gamma-2}}=0
\end{aligned}
$$

We transform the third term as follows:

$$
(r X-\psi) \beta_{t}\left(X-a_{t}\right)^{\gamma-1}=r \beta_{t}\left(X-a_{t}\right)^{\gamma}+\left(r a_{t}-\psi\right) \beta_{t}\left(X-a_{t}\right)^{\gamma-1}, \text { and }
$$

substitute in (33) to yield

$$
\begin{aligned}
& \beta_{t}^{\prime} \frac{\left(X-a_{t}\right)^{\gamma}}{\gamma}-\beta_{t} a_{t}^{\prime}\left(X-a_{t}\right)^{\gamma-1}+r \beta_{t}\left(X-a_{t}\right)^{\gamma}+\left(r a_{t}-\psi\right) \beta_{t}\left(X-a_{t}\right)^{\gamma-1} \\
& -\frac{1}{2} \frac{(\alpha-r)^{2}}{\sigma^{2}(\gamma-1)} \beta_{t}\left(X-a_{t}\right)^{\gamma}=0
\end{aligned}
$$

Separating (34) into terms in $\left(X-a_{t}\right)^{y}$ and $\left(X-a_{t}\right)^{\gamma-1}$ yields two equations

$$
\left.\begin{array}{l}
\text { [1] } \frac{\beta_{t}^{\prime}}{\gamma}+r \beta_{t}-\frac{1}{2} \beta_{t} \frac{(\alpha-r)^{2}}{\sigma^{2}(\gamma-1)}=0 \\
\text { [2] } a_{t}^{\prime}-r a_{t}+\psi=0
\end{array}\right\}
$$

Solutions of [1] and [2] with their limit conditions are

$$
\left.\begin{array}{l}
\beta_{t}=\exp \{\mu(T+N)\} \\
\text { with } \mu=-r\left\{r+\frac{(\alpha-r)^{2}}{2 \sigma^{2}(1-\gamma)}\right\} \\
\text { and } a_{t}=\psi\left\{\frac{1-e^{-r(T-N-t)}}{r}\right\}
\end{array}\right\}
$$

The function $a_{t}$ in (36) is the mathematical reserve required to meet the financial obligations of the pension fund manager using a discount rate, the risk-free rate. The term

$$
X-a_{t}=X-\psi\left\{\frac{1-e^{-r(T+N-t)}}{r}\right\}
$$

is the difference between the assets and liabilities.(see Pierre and Manuela (2003))

The above expression of (37) launches us into ALM scenario. We can then recast the value function as

$$
V(t, X)=e^{\mu(t-(T+N)} \frac{1}{\gamma}\left(X-a_{t}\right)^{\gamma}
$$

The optimal policy given by (30) yields

$$
\begin{aligned}
u_{t}^{*} & =\frac{\beta_{t}\left(X-a_{t}\right)^{\gamma-1}}{X \beta_{t}\left(\gamma-1\left(X-a_{t}\right)^{\gamma-2}\right.} \frac{\alpha-r}{\sigma^{2}} \\
& =\frac{\left(X-a_{t}\right)}{X} \frac{\alpha-r}{\sigma^{2}} \frac{1}{1-\gamma} \\
\Rightarrow & u_{t}^{*} X=\left(X-a_{t}\right) \frac{(\alpha-r)}{\sigma^{2}} \frac{1}{(1-\gamma)}
\end{aligned}
$$

$u_{t}^{*} X$ is the amount invested in risky asset, while the right hand side of (40) 
is the classical coefficient of Merton $\frac{(\alpha-r)}{\sigma^{2}} \frac{1}{(1-\gamma)}$ applied to a new process(see Merton, R.C.(1971))

$$
Z_{t}=X_{t}-a_{t}
$$

The process $Z_{t}$ represents the free surplus of the pension revolving fund, which is the difference between the asset and the discounted value of future obligations. This shows that at retirement age, the strategy should change so as to guarantee the new liability.

\section{OPTIMAL POLICY BEFORE RETIREMENT WHEN $\alpha=0$ AS IN THE CASE OF DEFINED BENEFIT CONTRIBUTION}

We are required to solve equation (8) when the contribution rate of the pensioner is zero $(\alpha=0)$

$$
\begin{aligned}
& \underset{u}{\operatorname{Max}} \operatorname{EU}(X(N)) \text { with } \\
& d X_{t}=X_{t}\left[\left(1-U_{t}\right) r\right] d t+X_{t} U_{t} \sigma d W_{t}, X(0)=p(0 \leq t \leq N)
\end{aligned}
$$

We state the value function of the problem as

$$
W(t, X)=\underset{u}{\operatorname{Max}} E[U(X(N)) \mid X(t)=X]
$$

with its associated HJB equation

$$
0=\max _{\{u\}}\left[\frac{\partial w}{\partial t}+\left(\left(r\left(1-u_{t}\right)\right) X \frac{\partial w}{\partial x}+\frac{1}{2} u_{t}^{2} \sigma^{2} X^{2} \frac{\partial^{2} w}{\partial x^{2}}\right]\right.
$$

The objective is to choose an allocation of the wealth so as to maximize the expected utility of the terminal wealth. We have

$$
V(t, x)=\operatorname{Sup}_{\mathbf{e}_{+}^{2}} E_{t, x}[U(X)]
$$

we the HJB equation associated with the problem and Uis the set of all admissible portfolios as a modified equation (44)

$$
\frac{\partial w}{\partial t}(t, x)+\operatorname{Sup}_{S \in R_{-}^{2}} L^{u} W(t, x)=0
$$

where $\mathrm{L}^{\mathrm{u}}$ is the $2^{\text {nd }}$ order linear operator

$$
L^{u} W(t, x)=\left(1-U_{t}\right) r X \frac{\partial w}{\partial x}(t, x)
$$

Hence we have the following two equations and a $2^{\text {nd }}$ order condition

$$
\left.\begin{array}{l}
\text { (a) } J\left(u_{t}^{*}\right)=0 \\
\text { (b) } \frac{\partial J}{\partial u}\left(u_{t}^{*}\right)=0 \\
\text { (c) } \frac{\partial^{2} J\left(u_{t}^{*}\right)}{\partial u^{2}}<0
\end{array}\right\}
$$

Substituting back into (44) we have

$$
0=-r X \frac{\partial w}{\partial x}+u_{t}^{*} X^{2} \sigma^{2} \frac{\partial^{2} w}{\partial x^{2}}
$$

and obtain a first explicit form for the optimal investment proportion ${ }^{u_{t}^{*}}$ in risky asset

$$
u_{t}^{*}=\frac{\partial w / \partial x}{X\left(\partial^{2} w / \partial x^{2}\right)} \square \frac{r}{\sigma^{2}}
$$


Substitute (50) into (48), we obtain a pde for the value function

$\frac{\partial w}{\partial t}+r X \frac{\partial w}{\partial x}-\frac{1}{2} \frac{(-r)^{2}}{\sigma^{2}} \frac{(\partial w / \partial x)^{2}}{\left(\partial^{2} w / \partial x^{2}\right)}=0$

i.e. $\frac{\partial w}{\partial t}+r X \frac{\partial w}{\partial x}-\frac{1}{2}\left(\frac{r}{\sigma}\right)^{2} \frac{(\partial w / \partial x)^{2}}{\left(\partial^{2} w / \partial x^{2}\right)}=0$

with condition $W(N, x)=U(x)$

Solving (51) for the value function $\mathrm{W}$ and substitute it in (50) to obtain the optimal policy before retirement when contribution rate is zero as in the case of defined benefit contribution. The equation admits some explicit solutions for the power law utility

$$
U(X)=\frac{X^{\gamma}}{\gamma}(\text { with } \gamma<1, \gamma \neq 0)
$$

The solution of $(51)$ is the same as the structure defined in (20b).

The differential equation for $\beta$ from (20) is

$$
\begin{aligned}
& \beta_{t}^{\prime}+r \gamma \beta_{t}+\beta_{t} \gamma \frac{(-r)^{2}}{2 \sigma^{2}(1-\gamma)}=0 \\
& \text { i.e. } \beta_{t}^{\prime}+r \gamma \beta_{t}+\beta_{t} \gamma \frac{r^{2}}{2 \sigma^{2}(1-\gamma)}=0
\end{aligned}
$$

The solution is given by

$$
\begin{aligned}
& \beta_{t}=e^{\mu(t-N)}, \\
& \text { where } \mu=-r\left[r+\frac{(-r)^{2}}{2 \sigma^{2}(1-\gamma)}\right] \\
& =-r\left[r+\frac{(r)^{2}}{2 \sigma^{2}(1-\gamma)}\right]=-r^{2}\left[1+\frac{r}{2 \sigma^{2}(1-\gamma)}\right]
\end{aligned}
$$

The $2^{\text {nd }}$ order condition is also satisfied

$\frac{\partial^{2} J}{\partial u^{2}}=X^{2} \sigma^{2} \frac{\partial^{2} w}{\partial X^{2}}=X^{2} \sigma^{2} e^{\mu(t-N)}(\gamma-1) X^{(\gamma-2)}<0$ the optimal proportion ${ }^{*}$ we obtain the explicit optimal policy:

$$
\begin{aligned}
U_{t}^{*} & =\frac{\beta_{t} X^{\gamma-1}}{X \beta_{t}(\gamma-1) X^{\gamma-2}} \frac{(-r)}{\sigma^{2}}, \\
U_{t}^{*} & =\frac{-r}{\sigma^{2}} \cdot \frac{1}{1-\gamma}=\frac{r}{\sigma^{2}(\gamma-1)}
\end{aligned}
$$

This is a constant proportion depending on the risk premium $(\alpha-r ; \alpha=0 \Rightarrow-r)_{\text {ithe volatility }} \sigma^{2}$ and the risk aversion ${ }^{\gamma}$ 


\section{OPTIMAL POLICY AFTER RETIREMENT WHEN $\alpha=0$, AS IN THE CASE OF DEFINED BENEFIT CONTRIBUTION}

We formulate the problem as

$\operatorname{Max} E U(X(N+T))$

Subject to $d X_{t}=\left\{X_{t}\left[\left(1-\mu_{t}\right) r-\psi\right]\right\} d t+X_{t} u_{t} \sigma d W_{t},(N \leq t \leq N+Y)$

where $X(N)$ is the amount obtained at retirement.

The value function of process (57) is defined by

$$
V(t, X)=\operatorname{Max}_{u} E\left\{U(X(N+T)) \mid X_{t}=X\right\}, N \leq t \leq N+T
$$

and the maximum principal gives

$$
\begin{aligned}
& 0=\operatorname{Max}_{u}\left\{\frac{\partial V}{\partial t}+\left[\left\{U_{t}(-r)+r\right\} X-\psi\right] \frac{\partial V}{\partial X}+\frac{1}{2} U_{t}^{2} \sigma^{2} X_{t}^{2} \frac{\partial^{2} W}{\partial X^{2}}\right\} \\
& \text { OR } \\
& 0=\operatorname{Max}_{u}\{\theta\}
\end{aligned}
$$

We obtain the following three conditions

$$
\left.\begin{array}{l}
\text { (a) } \theta u_{t}^{*}=0 \\
\text { (b) } \frac{\partial \theta}{\partial u}\left(u_{t}^{*}\right)=0 \\
\text { (c) } \frac{\partial^{2} \theta\left(u_{t}^{*}\right)}{\partial u^{2}}<0
\end{array}\right\}
$$

The same explicit form for the optimal control as in (50) is obtained

$$
u_{t}^{*}=-\frac{\partial V / \partial X}{X\left(\partial^{2} V / \partial X^{2}\right)} \frac{(-r)}{\sigma^{2}}=\frac{\partial V / \partial X}{X\left(\partial^{2} V / \partial X^{2}\right)}\left(\frac{r}{\sigma^{2}}\right)=0
$$

Plug back into (60) we obtain the pde

$$
\begin{aligned}
& \frac{\partial V}{\partial t}+\frac{\partial V}{\partial X}(r X-\psi)-\frac{1}{2} \frac{r^{2}(\partial V / \partial X)^{2}}{\sigma^{2}\left(\partial^{2} V / \partial X^{2}\right)}=0 \\
& \text { with } V(N+T, X)=U(X)
\end{aligned}
$$

We need to solve (62) for the value function Vand substitute it in (61) to obtain the optimal policy when contribution rate is zero.

This equation also admits some explicit solutions for power law utility of the form

$$
U(X)=\frac{X^{\gamma}}{\gamma}(\text { with } \gamma<1, \gamma \neq 0)
$$

We require solution of (62) with a candidate

$$
V(t, X)=\frac{\beta\left(X-a_{t}\right)^{\gamma}}{\gamma} \text { with limit conditions } a(N+T)=0, \beta(N+T)=1
$$

Where $\mathrm{a}_{\mathrm{t}} \mathrm{is}$ the risk free rate required to meet the future obligation of the fund manager.

Then,

$$
\begin{aligned}
& \frac{\partial V}{\partial t}=\beta_{t}^{\prime} \frac{\left(X-a_{t}\right)^{\gamma}}{\gamma}-\beta_{t}\left(X-a_{t}\right)^{\gamma-1} a_{t}, \\
& \frac{\partial V}{\partial X}=\beta_{t}\left(X-a_{t}\right)^{\gamma-1},
\end{aligned}
$$




$$
\frac{\partial^{2} V}{\partial X^{2}}=\beta_{t}(\gamma-1)\left(X-a_{t}\right)^{\gamma-2}
$$

Substitute back these derivatives in (62)

$$
\begin{aligned}
& \beta_{t}^{\prime} \frac{\left(X-a_{t}\right)^{\gamma}}{\gamma}-\beta_{t}\left(X-a_{t}\right)^{\gamma-1} a_{t}^{\prime}+(r X-\psi) \beta_{t}\left(X-a_{t}\right)^{\gamma-1} \\
& -\frac{1}{2} \frac{(-r)^{2} \beta_{t}^{2}\left(X-a_{t}\right)^{2(\gamma-1)}}{\sigma^{2} \beta_{t}(\gamma-1)\left(X-a_{t}\right)^{\gamma-2}}=0
\end{aligned}
$$

We transform the third term in (64) as follows:

$$
\begin{gathered}
(r X-\psi) \beta_{t}\left(X-a_{t}\right)^{\gamma-1}=r \beta_{t}\left(X-a_{t}\right)^{\gamma}+\left(r a_{t}-\psi\right) \beta_{t}\left(X-a_{t}\right)^{\gamma-1} \text { and substitute in (64) } \\
\beta_{t}^{\prime} \frac{\left(X-a_{t}\right)^{\gamma}}{\gamma}-\beta_{t} a_{t}^{\prime}\left(X-a_{t}\right)^{\gamma-1}+r \beta_{t}\left(X-a_{t}\right)^{\gamma}+\left(r a_{t}-\psi\right) \beta_{t}\left(X-a_{t}\right)^{\gamma-1} \\
-\frac{1}{2}\left(\frac{r}{\sigma}\right)^{2} \frac{\beta_{t}\left(X-a_{t}\right)^{\gamma}}{(\gamma-1)}=0
\end{gathered}
$$

Separating (65) into terms of $\left(X-a_{t}\right)^{y}$ and $\left(X-a_{t}\right)^{\gamma-1}$ yields two sets of equations

$$
\left.\begin{array}{l}
\text { [1] } \frac{\beta_{t}^{\prime}}{\gamma}+r \beta_{t}-\frac{1}{2} \beta_{t}\left(\frac{r}{\sigma}\right)^{2} \frac{1}{(\gamma-1)}=0 \\
\text { [2] } a_{t}^{\prime}-r a_{t}+\psi=0
\end{array}\right\}
$$

Solutions of [1] and [2] with their limit conditions are

$$
\begin{aligned}
& \beta_{t}=\exp \{\mu(T+N)\} \\
& \text { with } \mu=-r\left\{r+\frac{1}{2}\left(\frac{r}{\sigma}\right)^{2} \frac{1}{(1-\gamma)}\right\} \\
& \text { and } a_{t}=\psi\left\{\frac{1-e^{-r(T-N-t)}}{r}\right\}
\end{aligned}
$$

The function $\mathrm{a}_{\mathrm{t}}$ as previously defined is the required reserve to meet the financial obligations of the pension fund manager using a discount rate, the risk-free rate, when contribution rate is zero as in the case of defined benefit, where the rate of contribution by the employee is zero

The term

$$
X-a_{t}=X-\psi\left\{\frac{1-e^{-\xi}}{r}\right\} \text {, where } \xi=r(T+N-t)
$$

is the difference between the assets and the liabilities to be managed in the defined contribution settings.

Recasting the value function as

$$
V(t, X)=e^{\mu(t-(T+N))} \frac{\left(X-a_{t}\right)^{\gamma}}{\gamma}
$$

satisfying the $2^{\text {nd }}$ order conditions as

$$
\begin{aligned}
\frac{\partial^{2} \theta}{\partial u^{2}} & =X^{2} \sigma^{2} \frac{\partial^{2} V}{\partial X^{2}} \\
& =X^{2} \sigma^{2} \exp \{\mu(t-(T+N))\}(\gamma-1)\left(X-a_{t}\right)^{\gamma-2}<0
\end{aligned}
$$


The optimal policy given by (61) yields

$$
\begin{gathered}
u_{t}^{*}=\frac{\beta_{\mathrm{g}}\left(X-a_{\mathrm{g}}\right) \gamma-1}{X \beta_{\mathrm{t}}(\gamma-1)\left(X-a_{\mathrm{t}}\right) \gamma-2} \times \frac{(-\gamma)}{\sigma^{2}} \\
=\frac{\left(X-a_{\mathrm{g}}\right)}{x} \times \frac{\gamma}{\sigma^{2}} \times \frac{1}{\gamma-1} \\
u_{\mathrm{t}}^{*} X=\frac{\left(X-a_{\mathrm{g}}\right)}{\gamma-1} \times \frac{\gamma}{\sigma^{2}} .
\end{gathered}
$$

$u_{i}^{*} X \quad$ is the amount invested in risky asset in the defined benefit scheme, while the right hand side of (71) still remains the classical coefficient of Merton $\frac{r}{(1-\gamma) \sigma^{2}}$ applied to a process $Z_{t}=X_{t}-a_{t}$, which represents the free surplus of the pension revolving fund also is the difference between the asset and discounted value of fund manager's obligations.

\section{CONCLUSION}

We have examined herein the effect of liability on the optimal investment of the fund reserve in a defined contribution pension scheme as well as defined benefit pension scheme for before and after retirement.

\section{Before retirement}

For the defined benefit, $U_{t}^{*}=\frac{r}{\sigma^{2}(\gamma-1)^{*}}$ That is, the investment proportion is a constant proportion that depends on the interest rate $r$, the volatility $\sigma$ and $\gamma$ the risk aversion. Notice that $U_{t}^{*} \rightarrow 0$ as $r \rightarrow 0$; implying that the fund manager has no fund to invest with if the pensioner firm makes no contribution. This will affect the economic index of the pensioner's nation negatively.

On the other hand, as $r \rightarrow 0,, U_{t}^{*}=\frac{\alpha}{\sigma^{2}(\gamma-1)}$ for the defined contribution. Thus the economy of the pensioner as well as the economic index of his nation is positively affected since there always some contribution made by the pensioner irrespective of his firm's inability to contribution.

\section{After retirement}

For the defined benefit, $U_{t}^{*} X=\frac{r}{\sigma^{2}(\gamma-1)}\left(X-a_{t}\right)$. If $\gamma \leq 0$, we write (with brevity) $U_{t}^{*} X=\frac{r}{\sigma^{2}(\gamma-1)}\left(a_{t}-X\right)$. This implies that the amount required to meet the financial obligations of the pension fund manager must be high enough to exceed the wealth of the pensioner. This will in fact stress the reserve of the pensioner's firm and may lead to the liquidation of the firm- which will in turn, affect the economic index of the nation.

Notice also that $U_{t}^{*} \rightarrow 0$ as $r \rightarrow 0$ For the defined contribution; $U_{t}^{*} X=\frac{a}{\sigma^{2}(1-\gamma)}\left(X-a_{t}\right)$ as $r \rightarrow 0$, leaving a free surplus of the pension fund for the manager. We conclude that to meet the future financial obligations of the fund manager, the defined contribution scheme is to be adopted. This is the classical way to strategize as it decreases the amount invested in the risky assets (as seen by the pensioner's firm) so as to guarantee the needed liquidity.

We have examined the effect of liability on the optimal investment of the fund reserve in a defined contribution scheme. We conclude that the classical way to strategize this, is to decrease the amount invested in risky assets to guarantee the liquidity needed to meet the future financial obligations of the fund manger 


\section{REFERENCES}

[1]. Bjork, T. (1998). Arbitrage Theory in Continuous Time, Oxford University Press

[2]. Blake, D.(1998). Pension scheme as option on pension fund assets: Implications for pension fund management. Insurance: Mathematics and Economics 23, 263-286.

[3]. Booth, P., Yakonbov, Y., (2000). Investment for defined contribution pension scheme members close to retirement: An analysis of the "life style" concept. North American Actuarial Journal 4(2), 1-19.

[4]. Cairns, A.J.G.(2000). Some notes on the dynamics and optimal control of stochastic pension fund models in continuous time, ASTIN Bulletin 30: 19-55.

[5]. Constantinides, G. M.(1979). Multiperiod Consumption and Investment Behavior with Convex Transaction Cost .Management Science, 25, 1127- 1137.

[6]. Constantinides, G. M.(1986). Capital Market Equilibrium with Transaction Costs. Journal of Political Economy, 94, 842-862.

[7]. Fleming, W. H. (1995). Optimal models and risk- sensitive stochastic control. IMA Math. Appl. Vol. 65, 75-88.

[8]. Fleming, W. H. and Sheu, S. J. (1999). Optimal long term growth rate of expected utility of wealth. Ann. Appl. Probab. 9,871-903.

[9]. Deelstra, G., Grasselli, M.,Koehl, P.F.,(2002). Optimal design of the guarantee for defined contribution funds. Journal of Economic Dynamics and Control, Vol. 28, 2239-2260

[10]. Josa-Fombellida, R., Rincon-Zaratero, J.P.,(2010). Optimal asset allocation for aggregated defined benefit pension funds with stochastic interest rates. European journal of Operational Research, vol. 201, 211-221

[11]. Lim, Andrew E.B., Wong,B.,(2010). A benchmarking approach to the optimal asset allocation for insurers and pension funds. Insurance: Mathematics and Economics, vol. 46, 317-327

[12]. Merton, R.C. (1969). Lifetime portfolio selection under uncertainty: the continuous case. Review of Economics and Statistics 51, 247-257

[13]. Merton, R.C.(1971). Optimal consumption and portfolio rules in a continuous time model. Journal of Economic Theory 3:373-413

[14]. Osu, B.O. (2011). The price of asset-liability control under tail conditional expectation with no transaction cost. British Journal of Mathematics and Computer Science 1(3): 129-140.

[15]. Pierre, D., Manuela, B.P.,Immaculada, D.F.(2003). Stochastic optimal control of annuity contracts. Insurance: Mathematics and Economics Vol.33, 227-238.

[16]. Wang, H. and Wang, C(2010). Leverage management. Math. Finan. Econ. 3, 161-183. 